\title{
Contents
}

\section{Shell Structure of Exotic Nuclei}

T. Otsuka.................................... 1

1 Basics of Shell Model ............................. 1

2 Construction of an Effective Interaction and an Example in the $p f$ Shell ........................................ 16

3 The $N=20$ Problem: Does the Gap Change? .............. 18

4 Summary................................. 20

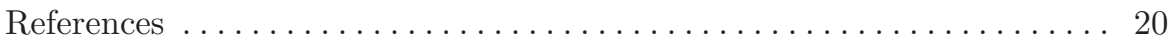

Testing the Structure of Exotic Nuclei via Coulomb Excitation of Radioactive Ion Beams at Intermediate Energies

T. Glasmacher ................................. 27

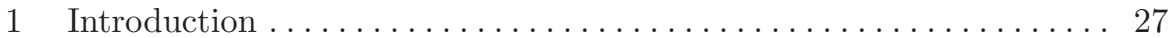

2 Experimental Considerations...................... 31

3 Extraction of Transition Matrix Elements from Cross Sections ..... 41

4 Recent Experimental Results . . . . . . . . .............. 43

5 Accuracy of the Technique......................... 48

6 Outlook and Summary ............................ 50

References ................................... 50

\section{Test of Isospin Symmetry Along the $N=Z$ Line}

S.M. Lenzi, M.A. Bentley............................ 57

1 Introduction ................................. 57

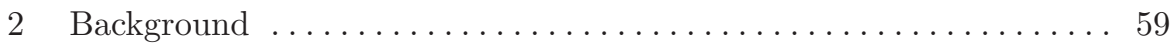

3 Experimental and Theoretical Tools .................. 67

4 Description of Excitation Energy Differences ................. 73

5 Isobaric Multiplets in the $f_{\frac{7}{2}}$ Shell .................... 84

6 Isobaric Multiplets in the $s d$ and $f p$ Shells................ 92

7 Conclusions and Outlook ............................... 95

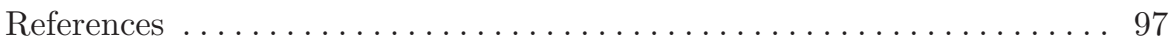




\section{Beta Decay of Exotic Nuclei}

B. Rubio and W. Gelletly ................................ 99

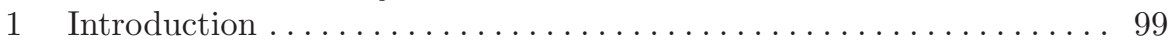

2 Beta Decay and Nuclear Structure ...................... 103

3 Experimental Considerations............................... 114

4 Some Illustrative Examples . . . . . . . . . . . . . . . . . . . . 122

5 Future Measurements . . . . . . . . . . . . . . . . . . . . . . . . 147

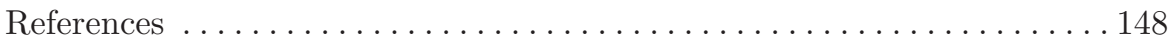

\section{One- and Two-Proton Radioactivity}

B. Blank.......................................... 153

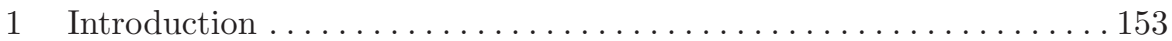

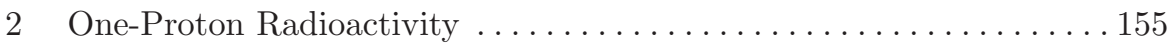

3 Two-Proton Radioactivity ............................... 174

4 Other Exotic Decay Channels .............................. 190

5 Conclusions.................................... 192

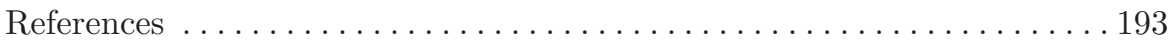

\section{Superheavy Elements}

S. Hofmann ........................................2203

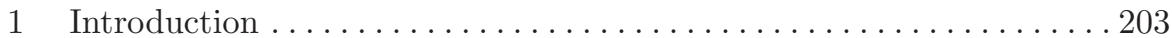

2 Experimental Techniques .................................. 205

3 Experimental Results ................................213

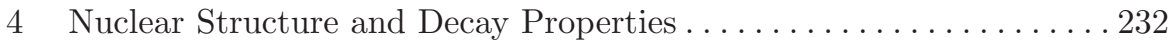

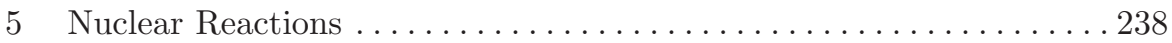

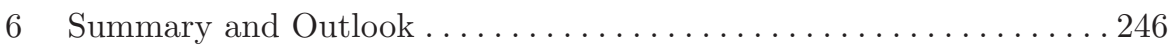

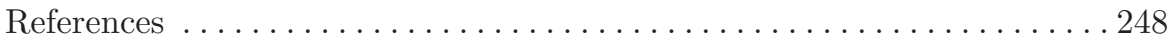

\section{Experimental Tools for Nuclear Astrophysics}

C. Angulo.......................................253

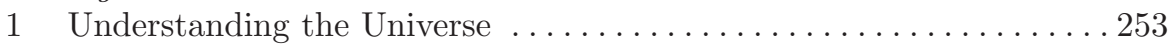

2 Relevant Quantities at Stellar Energies .................... 256

3 Stellar Cycles and Some Key Reactions .................. 260

4 Experimental Techniques in Nuclear Astrophysics .............. 262

5 Future Challenges and Conclusions . . . . . . . . . . . . . . . . 279

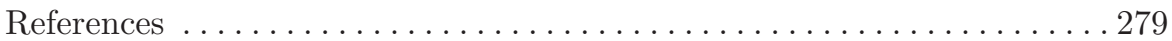

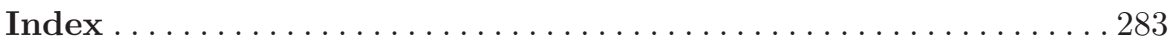

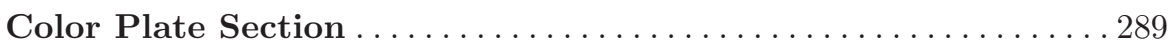

